\title{
Burnout in Ophthalmology Residency: A National Survey
}

\author{
Shu Feng, MD ${ }^{1}$ Parisa Taravati, MD ${ }^{1}$ Leona Ding, $\mathrm{MS}^{1} \quad$ Shivali Menda, MD ${ }^{1}$ \\ ${ }^{1}$ Department of Ophthalmology, University of Washington, Seattle, \\ Washington \\ J Acad Ophthalmol 2018;10:e98-e107. \\ Address for correspondence Shivali Menda, MD, Department of \\ Ophthalmology, University of Washington, Box 359608, 325 Ninth \\ Avenue, Seattle, WA 98104 (e-mail: shivali@uw.edu).
}

\begin{abstract}
Purpose To determine prevalence of burnout in ophthalmology residents in the United States using the Maslach Burnout Inventory-Human Services Survey and to identify factors associated with burnout and wellness.

Methods Anonymous survey distributed to residents via residency program directors.

Results Ninety-one programs out of 113 (80.5\%) confirmed survey distribution. Of 267 included respondents, 23 (8.6\%) met criteria for high burnout, and 169 (63.3\%) met criteria for at least one aspect of burnout. Longer work hours, longer hours spent on call, higher number of consults seen on call, fewer hours of sleep on call, and not meeting recommended physical activity minimums were associated with higher emotional exhaustion. Most common factors cited to improve wellness included

Keywords

- residency

- burnout

- wellness

- satisfaction physical activity, nurturing relationships, and co-resident support, whereas most commonly cited factors that contributed to burnout included sleep deprivation, call, and workload.

Conclusion Burnout in ophthalmology residents is common, occurring at similar rates as reported in residencies of other specialties.
\end{abstract}

Burnout is defined by a psychological syndrome of emotional exhaustion, depersonalization, and diminished sense of personal accomplishment. ${ }^{1}$ Burnout among medical residents has been a widely studied topic in the past few years, as it has been associated with higher rates of depression, ${ }^{2-5}$ higher risk for medical errors, ${ }^{6-10}$ higher rates of attrition, ${ }^{11,12}$ and poorer patient safety outcomes. ${ }^{6}$ While ophthalmology has traditionally been viewed as a "lifestyle specialty," the steep learning curve, frequent call, and technical demands of ophthalmic surgery are factors that may increase risk for burnout. A recent survey of ophthalmology program directors suggests that there is substantial burnout among ophthalmology residents, with $25 \%$ of respondents stating they faced an issue regarding resident depression, burnout, or suicide within the past year. $^{13}$

received

April 4, 2018

accepted after revision

June 4, 2018
DOI https://doi.org/

10.1055/s-0038-1666850.

ISSN $2475-4757$.
Burnout among physicians has been reported to be as high as $54.4 \%,{ }^{14}$ and residency and fellowship training is a period during which overall burnout, depersonalization, and fatigue are most prevalent. ${ }^{15}$ Recently, a national survey of burnout among general surgery trainees revealed that $69 \%$ of general surgery residents experience high burnout in at least one subscale. ${ }^{11}$ Similarly, past multi-institutional cross-sectional surveys in emergency medicine, ${ }^{16}$ anesthesia, ${ }^{6}$ obstetrics and gynecology, ${ }^{17}$ radiology, ${ }^{18,19}$ otolaryngology, ${ }^{20}$ neurosurgery, ${ }^{21}$ radiation, ${ }^{22}$ family medicine, ${ }^{2}$ and pediatrics ${ }^{23}$ reported burnout rates in residency training ranging from $33 \%$ (radiation) to $87 \%$ (otolaryngology).

This study aims to determine the prevalence of burnout among U.S. ophthalmology residents through a national survey and to associate burnout with demographic factors, year in training, practice setting, self-reported workload,

Copyright $\odot 2018$ by Thieme Medical Publishers, Inc., 333 Seventh Avenue, New York, NY 10001, USA. Tel: +1(212) 584-4662.
License terms

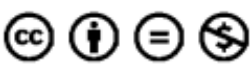


physical activity, and sleep. Additionally, this survey seeks to solicit comments from ophthalmology residents regarding factors that they personally felt to positively and negatively affect wellness and quality of life.

\section{Methods}

The University of Washington Institutional Review Board (IRB) ruled that the study was IRB exempt and did not require IRB approval. All ophthalmology residents in PGY-2 through PGY-4 years enrolled in a U.S. ophthalmology residency program were eligible to participate in this survey. A 46item electronic survey was distributed to all U.S. ophthalmology residency program directors and coordinators in January 2017. The recruitment email was forwarded to ophthalmology residents, who were eligible to enter a drawing to win a \$20 Amazon.com gift certificate. We asked programs to confirm participation and sent reminder emails. Two additional reminder emails were sent through the Association of University Professors of Ophthalmology (AUPO) email listserve and directly to program directors and coordinators. The survey was open between January 17 , 2017, and March 18, 2017.

Participation in the survey was anonymous and voluntary. The survey included 46 questions regarding sociodemographic factors, work hours, workload, sleep, burnout, and self-reported factors that affect wellness. Sociodemographic data included age, gender, marital status, and parent status. Work hour data collected included self-reported hours spent per week in clinic, operating room, didactics, research, consults, on-call duties, and study. Some questions addressed practice setting for the current rotation, call frequency, number of encounters typically seen on call, average sleep, average sleep on call, and hours of weekly physical activity. The survey also asked residents to rate their satisfaction with their specialty choice on a scale of 1 to 5 with 5 signifying very satisfied.

Burnout was measured using the 22-item Maslach Burnout Inventory-Human Services Survey (MBI-HSS), a validated questionnaire designed to measure burnout across three subscales of emotional exhaustion (EE), depersonalization (DP), and personal accomplishment (PA). ${ }^{1}$ Imputation was used to handle missing data and provide unbiased estimates and standard errors. Twenty subjects omitted one or two questions. One subject omitted more than two questions and was excluded from burnout analysis.

Total scores on the three subscales were divided into low, medium, or high tertiles based on previously validated normative data from the MBI-HSS manual (low emotional exhaustion: <17, moderate emotional exhaustion: 17-26; high emotional exhaustion: $>26$; low depersonalization: $<7$, moderate: depersonalization $7-12$, high depersonalization: $>12$; low personal accomplishment: $<32$, moderate personal accomplishment: $32-38$, and high personal accomplishment: $>38$ ). We defined burnout as meeting the criteria for burnout in at least one subscale: meeting the highest tertile category in emotional exhaustion, highest tertile category in depersonalization, or the lowest tertile category in personal accomplishment.

Association between categorical variables was determined by chi-squared testing. Association between continuous variables was determined by ANOVA testing. Given the exploratory nature of the data, $p$-values were not adjusted. Multiple imputations were performed using Statistical Package for the Social Sciences (SPSS) Version 24 with chained equations imputation (IBM Corporation, Armonk, New York, United States). All statistical analysis was conducted using MATLAB and Statistics Toolbox Release 2012b (The MathWorks, Inc., Natick, Massachusetts, United States) with $p<0.05$ considered statistically significant.

\section{Results}

Of the 113 programs contacted, 91 programs (80.5\%) replied to confirm participation. A total of 268 residents submitted the survey, representing $23 \%$ of 1,159 residents from the 91 programs that confirmed participation. Burnout data from one participant was excluded for omitting more than two questions on the MBI survey. Self-reported average work hour, sleep, and activity data are reported in - Table 1.

\section{Prevalence of Burnout}

One hundred sixty-nine of the 267 residents (63.3\%) that completed the survey met criteria for burnout in at least one subscale (-Fig. 1). One hundred forty-six of the 267 (54.7\%) residents met criteria for high emotional exhaustion, 124 of the 267 (46.4\%) met criteria for high depersonalization, and 35 of the 267 (13.1\%) met criteria for low personal accomplishment. Twenty-three of the 267 (8.6\%) met criteria of all three subscales (-Fig. 2 ).

Despite the high prevalence of burnout, average satisfaction with specialty choice was 4.2 out of 5 , with $81 \%$ of residents reporting satisfaction score of 4 or higher, and only $5.2 \%$ reporting a satisfaction of 2 or lower.

\section{Sociodemographic Factors Associated with Burnout}

- Table 2 demonstrates the impact of sociodemographic factors on scoring highly in at least one aspect of burnout and - Tables 3-5 display these associations with scores in each individual subscale of burnout. There is no association between gender, age, or year of clinical training and burnout in any category. Being married or in a long-term partnership is associated with lower levels of depersonalization $(p=0.03)$ and higher levels of personal accomplishment ( $p=0.03)$. Being a parent is associated with lower depersonalization $(p=0.03)$. Residents on consult or county rotations also reported higher depersonalization compared with those on academic, community, or Veterans' Affairs rotations ( $p=0.009$ ).

\section{Work Hours, Sleep, and Call Intensity}

Longer work hours are associated with burnout in at least one subcategory $(p=0.01)$ and higher levels of emotional exhaustion $(p=0.01)$. Specifically, more hours per week spent on consults is associated with burnout in at least one subcategory ( $p=0.04$ ), with higher levels of emotional 
Table 1 Average participant characteristics

\begin{tabular}{|l|l|l|l|}
\hline Characteristic & Mean & Range & Standard deviation \\
\hline Age & 29.7 & $25-42$ & 2.3 \\
\hline Sleep per night (h) & 6.7 & $<3-8$ & 1.6 \\
\hline Sleep on call per night (h) & 4.7 & $0-7$ & 1.6 \\
\hline Encounters per half day & 11.5 & $0-40$ & 6.5 \\
\hline Weekday night call encounters & 3.2 & $0-30$ & 3.5 \\
\hline Weekly work hours & 67 & $14-166$ & 17 \\
\hline Hours on consults per week (excl. call) & 4.8 & $0-70$ & 11.0 \\
\hline Hours on call per week & 9.9 & $0-62$ & 9.8 \\
\hline Hours studying per week & 8.9 & $0-36$ & 6.2 \\
\hline Weekly physical activity (h) & 2.1 & $0-8$ & 1.9 \\
\hline Satisfaction with specialty choice (1-5 scale) & 4.2 & $1-5$ & 0.9 \\
\hline
\end{tabular}

${ }^{a}$ Excluding two extreme outliers of 45 and 82 hours per week.

exhaustion $(p=0.01)$ and depersonalization $(p=0.02)$. Those who reported more hours spent on call were more likely to experience burnout in at least one subcategory $(p=0.003)$ and higher emotional exhaustion $(p=0.007)$. Similarly, higher number of encounters reported for an average weekday call night are associated with emotional exhaustion $(p=0.01)$ and depersonalization $(p=0.02)$. Those who scored high in at least one subcategory of burnout reported fewer hours of sleep on call $(p=0.001)$, with a strong association between emotional exhaustion and amount of sleep on call ( $p=0.00003$ ). Overall, average nightly sleep was not associated with any subset of burnout.

\section{Physical Activity}

Reported physical activity per week ranged from 0 to 8 hours a week (excluding two extreme outliers of 45 and 82 hours which appeared to be due to incorrectly entered or measured data, as it appeared unlikely for a resident to average more than 40 hours a week of physical activity). When dividing residents between those with less than 150 minutes per week (defined as minimum recommended physical activity weekly per the Department of Health and Human Services) and those with 150 minutes per week or greater of physical activity, there was an association between meeting recommended weekly physical activity and less emotional exhaustion $(p=0.02)$.

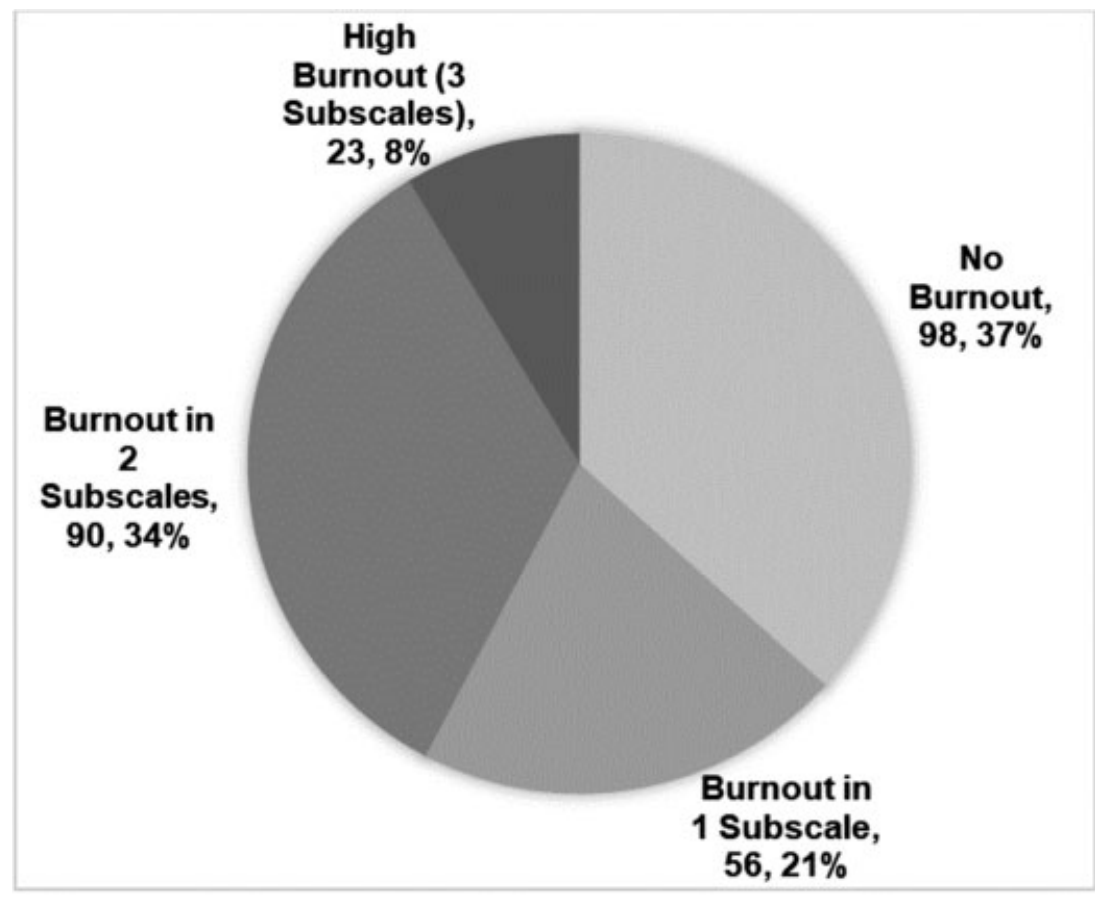

Fig. 1 Percentage of residents with high burnout in $0,1,2$, and 3 subsets. 

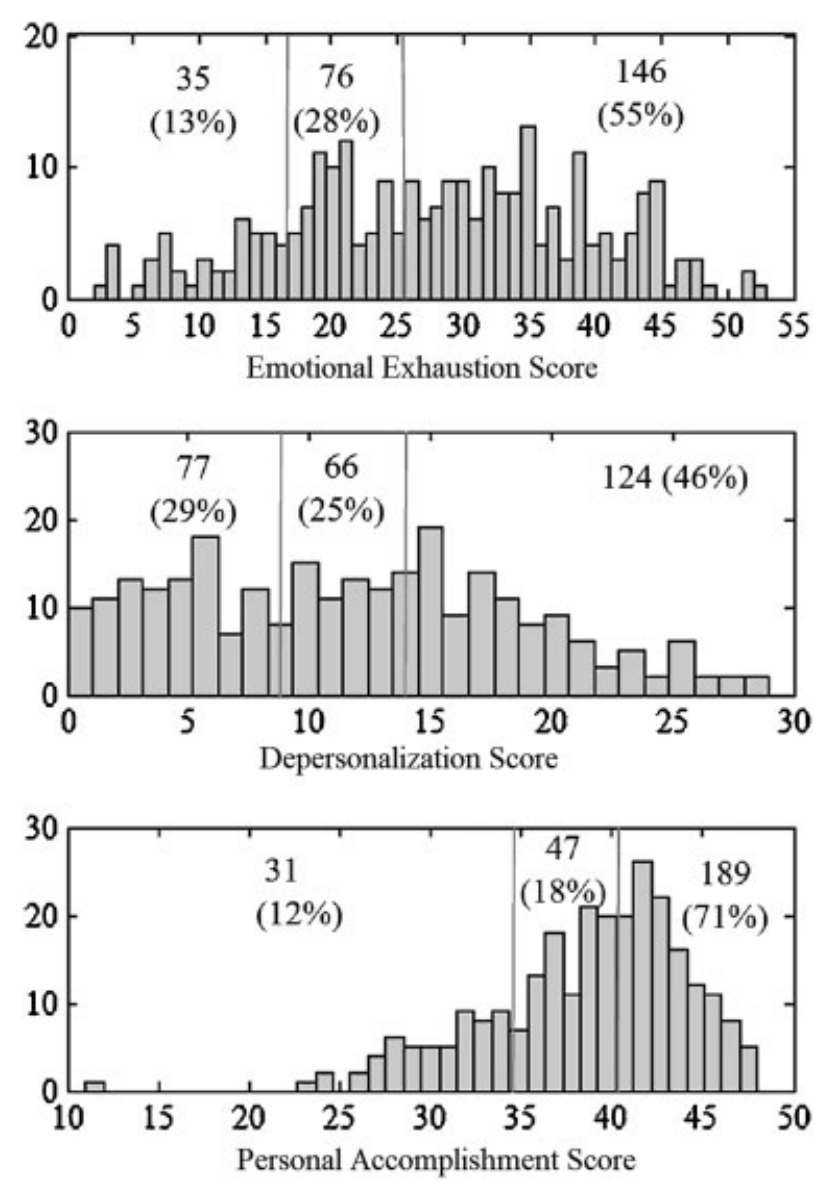

Fig. 2 Histograms of emotional exhaustion, depersonalization, and personal accomplishment scores illustrating number and percentage of residents scoring in each tertile (low, medium, high). High burnout is a scoring in the highest tertile for emotional exhaustion and the lowest tertile in personal accomplishment.

\section{Self-Reported Factors Related to Wellness}

- Table 6 lists the most commonly cited factors that positively or negatively affect well-being with the number of times these factors were reported in the free response section. Among the reported factors that increased wellness, the majority of residents (194) cited relationships with family or friends. Many also cited physical activity (129) and relationship with co-residents (74).

Regarding factors that negatively affect well-being, poor sleep was most commonly mentioned (by 74 residents). Similarly, call duties were noted as being detrimental to well-being by 69 residents, with 11 residents citing the lack of protection for home-call and need to return to clinic for a full day post-call as being additional stressors. Fifty-nine residents reported workload or work obligations as harmful to their well-being, and in particular over-booked clinics and nonclinical/administrative duties that residents considered noneducational.

Thirty-five residents similarly reported that wellness was hindered by long work hours. They felt that they did not have time outside of work to participate in leisurely activities that improve wellness or to interact with family outside of work (47). Additionally, 33 residents cited the need to study and lack of study time as a stressor, as well as pressure to perform well on the Ophthalmic Knowledge Assessment Program (OKAP). Several residents also reported poor exercise (16) or diet (16) as factors.

Thirty-one residents noted that poor faculty interaction was detrimental to their wellness due to high expectations or criticism for mistakes in the setting of a steep learning curve. Residents expressed that they felt underappreciated for their work and efforts to learn, and instead were criticized for errors. Other residents (32) reported positive faculty support as a major factor that improved well-being.

Twenty residents also cited the burden of conflicting time demands of residency, including the expectation to pursue outside research, journal clubs, and other required presentations in addition to clinical demands and studying. Many stated that there was no dedicated time for these other pursuits, leading to continued workload beyond work hours.

\section{Discussion}

The prevalence of burnout in ophthalmology residency at $63.3 \%$ is high considering the impact of burnout on resident wellness, attitudes toward patients, and patient care. However, these numbers are lower than a national survey of general surgery in 2016, in which 69\% experienced burnout. ${ }^{11}$ - Table 7 illustrates results of this study compared with multi-institutional studies in other specialties. Overall, compared with previous studies of other specialties, ophthalmology residents in this study experienced higher emotional exhaustion and depersonalization, but also had higher feelings of personal accomplishment. The percentage of residents with high burnout (burnout in all three subscales) is similar in our study compared with previous studies. ${ }^{11,17,20}$

Most demographic factors have no association with burnout, with the exception of being married or in a long-term partnership, which is associated with less depersonalization and higher personal accomplishment. Having children is also associated with less depersonalization. Marriage and parent status may serve as markers for built-in outside relationships, as the majority of residents also cited relationships with family and friends as beneficial to their well-being.

In addition to outside relationships, physical activity is also cited to be beneficial to quality of life by more than $50 \%$ of survey participants, and receiving more than the recommended 150 minutes of weekly exercise is associated with lower emotional exhaustion. These findings are similar to a recent study of internal medicine residents that found that residents able to meet physical activity guidelines were less likely to be burned out. ${ }^{24}$ Whether this relates to the positive effect of physical activity or the ability of residents to take time in their schedules to exercise is less certain; those who are able to make time for physical activity are presumably working less or have fewer outside demands on their time.

Increased work hours and call duties were also found to be associated with burnout, similar to several previous studies. ${ }^{11,25}$ While there is conflicting data regarding the effect of 2003 Accreditation Council for Graduate Medical 
e102 Burnout in Ophthalmology Residency Feng et al.

Table 2 Variables associated with burnout in any subset

\begin{tabular}{|c|c|c|c|c|}
\hline \multirow[b]{2}{*}{ Variable } & \multicolumn{3}{|c|}{ High score in any subset of burnout } & \multirow[b]{2}{*}{$p$-Value $\left(x^{2}\right)$} \\
\hline & Total & No & Yes & \\
\hline Total & & 98 & 169 & \\
\hline Gender, $n(\%)$ & & & & 0.45 \\
\hline Men & 142 & $56(37 \%)$ & $86(63 \%)$ & \\
\hline Women & 123 & $43(35 \%)$ & $80(65 \%)$ & \\
\hline Unknown & 2 & - & - & \\
\hline Relationship status, $n$ (\%) & & & & 0.096 \\
\hline Single & 83 & $25(30 \%)$ & $58(70 \%)$ & \\
\hline Partnered or married & 184 & $75(41 \%)$ & $109(59 \%)$ & \\
\hline Has children, $n(\%)$ & & & & 0.96 \\
\hline Yes & 53 & $20(38 \%)$ & $33(62 \%)$ & \\
\hline No & 214 & $80(37 \%)$ & $134(63 \%)$ & \\
\hline \multicolumn{2}{|c|}{ Year of clinical training, $n(\%)$} & & & 0.37 \\
\hline 2 & 108 & $35(32 \%)$ & $73(68 \%)$ & \\
\hline 3 & 89 & $36(40 \%)$ & $53(60 \%)$ & \\
\hline 4 & 70 & $29(41 \%)$ & 41 (59\%) & \\
\hline Rotation setting, $n(\%)$ & & & & 0.27 \\
\hline Academic & 155 & $64(41 \%)$ & 91 (59\%) & \\
\hline Community & 8 & $3(38 \%)$ & $5(62 \%)$ & \\
\hline Veterans' Affairs & 53 & $21(40 \%)$ & $32(60 \%)$ & \\
\hline Consult & 19 & $5(26 \%)$ & $14(74 \%)$ & \\
\hline County & 28 & $6(21 \%)$ & $22(79 \%)$ & \\
\hline Other & 4 & - & - & \\
\hline Physical activity, $n$ (\%) & & & & 0.13 \\
\hline$\geq 150$ min weekly & 94 & $41(44 \%)$ & $53(56 \%)$ & \\
\hline \multirow[t]{2}{*}{$<150$ min weekly } & 173 & $59(34 \%)$ & $114(66 \%)$ & \\
\hline & & No & Yes & $p$-Value (ANOVA) \\
\hline \multicolumn{2}{|l|}{ Age, mean (SD) } & $29.6(3.9)$ & $29.3(3.9)$ & 0.55 \\
\hline \multicolumn{2}{|c|}{ Estimated nightly sleep, mean (SD) } & $6.7(0.7)$ & $6.6(0.8)$ & 0.34 \\
\hline \multicolumn{2}{|c|}{ Estimated sleep on call, mean (SD) } & $5.1(1.6)$ & $4.4(1.6)$ & $0.0006^{\mathrm{a}}$ \\
\hline \multicolumn{2}{|c|}{ Encounters on weekday call night, mean (SD) } & $2.8(3.8)$ & $3.5(3.3)$ & 0.10 \\
\hline \multicolumn{2}{|c|}{ Work hours, mean (SD) } & $64.0(16.0)$ & $69.8(18.2)$ & $0.0096^{\mathrm{a}}$ \\
\hline \multicolumn{2}{|l|}{ On call hours, mean (SD) } & $7.5(8.6)$ & $11.2(10.3)$ & $0.0026^{a}$ \\
\hline \multicolumn{2}{|c|}{ Consults hours, mean (SD) } & $3.0(7.2)$ & $5.9(12.6)$ & $0.037^{\mathrm{a}}$ \\
\hline
\end{tabular}

${ }^{a}$ Statistically significant $(p<0.05)$.

Education (ACGME) duty hour restrictions on patient care and educational experience, these work hour reductions have been found to have improved resident well-being. ${ }^{26}$ In addition, call for ophthalmology residents can be high given the relatively small program sizes and tendency of residency programs to be based in tertiary referral centers. The structure and intensity of call varies significantly among programs, and these differences are not captured by our survey. However, there is a significant association in our study between intensity of call (assessed by number of average encounters on call and amount of sleep on call) and emotional exhaustion. Several residents cited the lack of post-call relief as a major detrimental factor to well-being. Some programs have, therefore, instituted in-house call, night-float, or post-call relief systems to alleviate this.

This study, as in all cross-sectional anonymous surveys, has major limitations due to survey bias. Those who fill out the survey are more likely to have an interest in burnout and therefore may be more likely to be burned out than nonresponders. Alternatively, some residents may be too burned 
Table 3 Variables associated with emotional exhaustion

\begin{tabular}{|c|c|c|c|c|c|}
\hline \multirow{2}{*}{ Variable } & \multicolumn{4}{|c|}{ Emotional exhaustion score } & \multirow[b]{2}{*}{$p$-Value $\left(x^{2}\right)$} \\
\hline & Total & Low & Medium & High & \\
\hline Gender, $n(\%)$ & & & & & 0.36 \\
\hline Men & 142 & $25(17.6 \%)$ & $45(31.6 \%)$ & $72(50.7 \%)$ & \\
\hline Women & 123 & $19(15.4 \%)$ & $31(25.2 \%)$ & $73(59.3 \%)$ & \\
\hline Unknown & 2 & - & - & & \\
\hline Relationship status, $n(\%)$ & & & & & 0.24 \\
\hline Single & 83 & $10(12.0 \%)$ & $22(26.5 \%)$ & $51(61.4 \%)$ & \\
\hline Partnered or married & 184 & $25(13.6 \%)$ & $54(29.3 \%)$ & $95(51.7 \%)$ & \\
\hline Has children, $n(\%)$ & & & & & 0.62 \\
\hline Yes & 53 & $11(20.8 \%)$ & $13(24.5 \%)$ & $29(54.7 \%)$ & \\
\hline No & 214 & $34(14.9 \%$ & $63(29.4 \%$ & $117(54.7 \%)$ & \\
\hline \multicolumn{2}{|c|}{ Year of clinical training, $n(\%)$} & & & & 0.47 \\
\hline 2 & 108 & $14(13.0 \%)$ & $28(25.9 \%)$ & $66(61.1 \%)$ & \\
\hline 3 & 89 & $17(19.1 \%)$ & $28(31.5 \%)$ & $44(49.4 \%)$ & \\
\hline 4 & 70 & $14(20.0 \%)$ & $20(28.6 \%)$ & $36(51.4 \%)$ & \\
\hline \multicolumn{2}{|l|}{ Rotation setting, $n(\%)$} & & & & 0.35 \\
\hline Academic & 155 & $27(17.4 \%)$ & $51(32.9 \%)$ & $77(49.7 \%)$ & \\
\hline Community & 8 & $2(25 \%)$ & $2(25 \%)$ & $4(50 \%)$ & \\
\hline Veterans' Affairs & 53 & $11(20.8 \%)$ & $13(24.5 \%)$ & $29(54.7 \%)$ & \\
\hline Consult & 19 & $2(10.5 \%)$ & $3(15.8 \%)$ & $14(73.6 \%)$ & \\
\hline County & 28 & $3(10.3 \%)$ & $5(17.9 \%)$ & $20(71.4 \%)$ & \\
\hline Other & 4 & - & - & - & \\
\hline \multicolumn{2}{|l|}{ Physical activity, $n$ (\%) } & & & & $0.020^{\mathrm{a}}$ \\
\hline$\geq 150$ min weekly & 94 & $24(25.5 \%)$ & $23(24.5 \%)$ & $47(50 \%)$ & \\
\hline \multirow[t]{2}{*}{$<150$ min weekly } & 173 & $21(13.9 \%)$ & $53(30.6 \%)$ & 99 (57.2\%) & \\
\hline & & & & & $p$-Value (ANOVA) \\
\hline \multicolumn{2}{|l|}{ Age, mean (SD) } & $29.1(4.9)$ & $29.9(2.7)$ & $29.3(4.1)$ & 0.21 \\
\hline \multicolumn{2}{|c|}{ Estimated nightly sleep, mean (SD) } & $6.8(0.7)$ & $6.6(0.7)$ & $6.6(0.8)$ & 0.12 \\
\hline \multicolumn{2}{|c|}{ Estimated sleep on call, mean (SD) } & $5.7(1.4)$ & $4.7(1.6)$ & $4.4(1.6)$ & $0.000033^{\mathrm{a}}$ \\
\hline \multicolumn{2}{|c|}{ Encounters on weekday call night, mean (SD) } & $1.8(1.8)$ & $3.3(4.2)$ & $3.6(3.4)$ & $0.014^{\mathrm{a}}$ \\
\hline \multicolumn{2}{|c|}{ Work hours, mean (SD) } & $61.9(13.4)$ & $65.0(16.1)$ & $70.8(18.9)$ & $0.010^{\mathrm{a}}$ \\
\hline \multicolumn{2}{|l|}{ On call hours, mean (SD) } & $7.4(10.8)$ & $7.6(7.1)$ & $11.7(10.4)$ & $0.0066^{\mathrm{a}}$ \\
\hline \multicolumn{2}{|c|}{ Consults hours, mean (SD) } & $2.0(3.3)$ & $3.3(8.1)$ & $6.4(13.3)$ & $0.013^{\mathrm{a}}$ \\
\hline
\end{tabular}

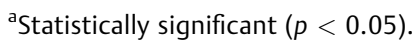

out to respond to a survey. Bias could also have introduced as the survey was available for participation only between January and March of 2017, which can be a particularly difficult time of year for residents due to the timing of the OKAP exam and the winter season. Additionally, survey answers were self-reported, and it is possible that residents who are experiencing burnout would self-report lower amount of sleep or higher numbers of hours worked.

Our respondents account for approximately $23 \%$ of ophthalmology residents. Our study, like other survey studies, had difficulty increasing the number of respondents. The studies done by Visser et al, ${ }^{27}$ Holbrook et al, ${ }^{28}$ and Mealing et al ${ }^{29}$ have concluded that studies with lower response rates have only marginally decreased demographic representativeness compared with those with higher response rates. Recruitment was also limited because survey information and reminders were sent through program directors and program coordinators rather than directly to residents. The survey also did not ask about specific program characteristics to protect the anonymity of programs and residents, but it is clear from survey responses that institution-specific cultural factors and aspects of program structure can strongly affect resident well-being. 
e104 Burnout in Ophthalmology Residency Feng et al.

Table 4 Variables associated with depersonalization

\begin{tabular}{|c|c|c|c|c|c|}
\hline \multirow[b]{2}{*}{ Variable } & \multicolumn{4}{|c|}{ Depersonalization score } & \multirow[b]{2}{*}{$p$-Value $\left(x^{2}\right)$} \\
\hline & Total & Low & Medium & High & \\
\hline & & 77 & 66 & 124 & \\
\hline Gender, $n$ (\%) & & & & & 0.32 \\
\hline Men & 142 & $38(26.7 \%)$ & $32(22.5 \%)$ & $72(50.7 \%)$ & \\
\hline Women & 123 & $38(30.9 \%)$ & $34(27.6 \%)$ & $51(41.5 \%)$ & \\
\hline Unknown & 2 & & & & \\
\hline Relationship status, $n(\%)$ & & & & & $0.033^{a}$ \\
\hline Single & 83 & $18(21.7 \%)$ & $17(21.7 \%)$ & $48(57.8 \%)$ & \\
\hline Partnered or married & 184 & $59(32.1 \%)$ & 49 (26.6\%) & $76(41.3 \%)$ & \\
\hline Has children, $n(\%)$ & & & & & $0.032^{a}$ \\
\hline Yes & 53 & $23(49.1 \%)$ & $10(18.9 \%)$ & $20(37.8 \%)$ & \\
\hline No & 214 & $54(25.2 \%)$ & $56(26.2 \%)$ & $104(48.6 \%)$ & \\
\hline \multicolumn{2}{|c|}{ Year of clinical training, $n(\%)$} & & & & 0.55 \\
\hline 2 & 108 & $28(25.9 \%)$ & $24(22.2 \%)$ & $56(51.8 \%)$ & \\
\hline 3 & 89 & $29(32.5 \%)$ & $21(23.6 \%)$ & $39(43.8 \%)$ & \\
\hline 4 & 70 & $20(28.6 \%)$ & $21(30 \%)$ & $29(41.4 \%)$ & \\
\hline \multicolumn{2}{|l|}{ Rotation setting, $n(\%)$} & & & & $0.0087^{a}$ \\
\hline Academic & 155 & $53(34.2 \%)$ & $34(21.9 \%)$ & $68(43.9 \%)$ & \\
\hline Community & 8 & $2(25 \%)$ & $4(50 \%)$ & $2(25 \%)$ & \\
\hline Veterans' Affairs & 53 & $15(28.3 \%)$ & $18(34.0 \%)$ & $20(37.7 \%)$ & \\
\hline Consult & 19 & $2(15.8 \%)$ & $7(42.1 \%)$ & $10(52.6 \%)$ & \\
\hline County & 28 & $4(14.3 \%)$ & $3(10.7 \%)$ & $21(75 \%)$ & \\
\hline Other & 4 & - & - & & \\
\hline \multicolumn{2}{|l|}{ Physical activity, $n$ (\%) } & & & & 0.32 \\
\hline$\geq 150$ min weekly & 94 & $29(30.9 \%)$ & $27(28.7 \%)$ & $38(40.4 \%)$ & \\
\hline \multirow[t]{2}{*}{$<150$ min weekly } & 173 & $48(27.7 \%)$ & 39 (22.5\%) & $86(49.7 \%)$ & \\
\hline & & & & & p-Value (ANOVA) \\
\hline \multicolumn{2}{|l|}{ Age, mean (SD) } & $30.0(4.5)$ & $29.5(1.9)$ & $29.0(4.2)$ & 0.21 \\
\hline \multicolumn{2}{|c|}{ Estimated nightly sleep, mean (SD) } & $6.6(0.8)$ & $6.7(0.8)$ & $6.6(0.7)$ & 0.91 \\
\hline \multicolumn{2}{|c|}{ Estimated sleep on call, mean (SD) } & $4.9(1.7)$ & $4.6(1.9)$ & $4.6(1.4)$ & 0.42 \\
\hline \multicolumn{2}{|c|}{$\begin{array}{l}\text { Encounters on weekday call night, } \\
\text { mean (SD) }\end{array}$} & $2.3(2.0)$ & $3.6(4.4)$ & $3.6(3.6)$ & $0.022^{\mathrm{a}}$ \\
\hline \multicolumn{2}{|l|}{ Work hours, mean (SD) } & $64.8(13.1)$ & 66.3 (18.9) & $70.0(19.1)$ & 0.093 \\
\hline \multicolumn{2}{|l|}{ On call hours, mean (SD) } & $8.3(9.9)$ & $8.7(10.6)$ & $11.3(9.3)$ & 0.066 \\
\hline \multicolumn{2}{|c|}{ Consults hours, mean (SD) } & $1.9(3.8)$ & $6.2(13.3)$ & $5.8(12.2)$ & $0.021^{\mathrm{a}}$ \\
\hline
\end{tabular}

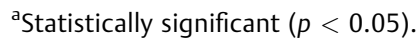

Despite these limitations, this survey offers insight into a range of factors that residents feel are important to wellbeing, and acknowledging burnout among ophthalmology residents may be the first step toward improving resident wellness.

Ultimately, the call and workload associated with burnout are a vital part of residency training because they provide crucial experiences during a limited period of training in a surgical field. Still, the steep learning curve of ophthalmol- ogy in combination with high work hours, intense call experiences, and minimal time for sleep and recovery can be naturally conducive to emotional exhaustion. Some burnout may be inevitable to residency; training stimulates residents to reach their maximum potential in the naturally uncomfortable position of new learners. For residents, it is important that their efforts are appreciated. Residency should be recognized as a period of vulnerability to burnout, and it appears from the subjective responses in our survey 
Table 5 Variables associated with personal accomplishment

\begin{tabular}{|c|c|c|c|c|c|}
\hline \multirow[b]{2}{*}{ Variable } & \multicolumn{4}{|c|}{ Personal accomplishment score } & \multirow[b]{2}{*}{$p$-Value $\left(x^{2}\right)$} \\
\hline & Total & Low & Medium & High & \\
\hline & & 31 & 47 & 189 & \\
\hline Gender, $n(\%)$ & & & & & 0.68 \\
\hline Men & 142 & $18(12.7 \%)$ & $22(15.5 \%)$ & $102(71.8 \%)$ & \\
\hline Women & 123 & $13(10.6 \%)$ & $24(19.5 \%)$ & $86(69.9 \%)$ & \\
\hline Unknown & 2 & & & & \\
\hline \multicolumn{2}{|l|}{ Relationship status, $n(\%)$} & & & & $0.034^{a}$ \\
\hline Single & 83 & $12(14.5 \%)$ & $21(25.3 \%)$ & $50(60.2 \%)$ & \\
\hline Partnered or married & 184 & $19(10.3 \%)$ & $26(14.1 \%)$ & $139(75.5 \%)$ & \\
\hline Has children, $n(\%)$ & & & & & 0.86 \\
\hline Yes & 53 & $6(11.3 \%)$ & $8(15.1 \%)$ & $39(73.6 \%)$ & \\
\hline No & 214 & $25(12.1 \%)$ & $39(18.2 \%)$ & $150(69.8 \%)$ & \\
\hline \multicolumn{2}{|c|}{ Year of clinical training, $n(\%)$} & & & & 0.62 \\
\hline 2 & 108 & $16(14.8 \%)$ & $21(19.4 \%)$ & $71(65.7 \%)$ & \\
\hline 3 & 89 & $8(9.0 \%)$ & $15(16.8 \%)$ & $66(74.2 \%)$ & \\
\hline 4 & 70 & $7(10 \%)$ & $11(15.7 \%)$ & $52(74.3 \%)$ & \\
\hline \multicolumn{2}{|l|}{ Rotation setting, $n(\%)$} & & & & 0.23 \\
\hline Academic & 155 & $19(12.3 \%)$ & $29(18.7 \%)$ & 107 (69.0\%) & \\
\hline Community & 8 & $0(0 \%)$ & $1(12.5 \%)$ & 7 (87.5\%) & \\
\hline Veterans' Affairs & 53 & $3(5.7 \%)$ & $7(13.2 \%)$ & $44(83.0 \%)$ & \\
\hline Consult & 19 & $1(5.3 \%)$ & $6(31.6 \%)$ & $12(78.9 \%)$ & \\
\hline County & 28 & $6(21.4 \%)$ & $4(14.3 \%)$ & $18(64.3 \%)$ & \\
\hline Other & 4 & - & - & & \\
\hline \multicolumn{2}{|l|}{ Physical activity, $n$ (\%) } & & & & 0.29 \\
\hline$\geq 150$ min weekly & 94 & $8(8.5 \%)$ & $14(14.9 \%)$ & $72(76.6 \%)$ & \\
\hline \multirow[t]{2}{*}{$<150$ min weekly } & 173 & $23(13.3 \%)$ & $33(19.1 \%)$ & $117(67.6 \%)$ & \\
\hline & & & & & $p$-Value (ANOVA) \\
\hline \multicolumn{2}{|l|}{ Age, mean (SD) } & $29.8(2.3)$ & $29.4(4.0)$ & $29.3(4.1)$ & 0.59 \\
\hline \multicolumn{2}{|c|}{ Estimated nightly sleep, mean (SD) } & $6.6(0.8)$ & $6.7(0.8)$ & $6.6(0.7)$ & 0.92 \\
\hline \multicolumn{2}{|c|}{ Estimated sleep on call, mean (SD) } & $6.7(0.7)$ & $6.5(0.8)$ & $6.6(0.7)$ & 0.54 \\
\hline \multicolumn{2}{|c|}{$\begin{array}{l}\text { Encounters on weekday call night, } \\
\text { mean (SD) }\end{array}$} & $4.5(1.5)$ & $4.6(1.6)$ & $4.8(1.6)$ & 0.41 \\
\hline \multicolumn{2}{|l|}{ Work hours, mean (SD) } & $64.8(13.1)$ & $66.3(18.9)$ & $70.0(19.1)$ & 0.16 \\
\hline \multicolumn{2}{|c|}{ On call hours, mean (SD) } & $11.9(9.8)$ & $10.5(11.3)$ & $9.0(9.0)$ & 0.28 \\
\hline \multicolumn{2}{|c|}{ Consults hours, mean (SD) } & $5.4(9.2)$ & $4.9(11.5)$ & $4.6(11.1)$ & 0.94 \\
\hline
\end{tabular}

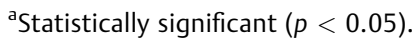

that cultural factors and attitudes toward trainees among institutions can significantly impact the residency training experience.

Many factors that influence burnout are not directly related to residency, instead to involving support systems, personal resilience, and outside interests. ${ }^{30-33}$ However, programs can provide a supportive environment by encouraging faculty to be understanding and supportive of resident efforts and to foster camaraderie between co-residents. For example, formal and informal mentorship has been shown in previous studies to be correlated with lower burnout among surgery residents. ${ }^{11,21}$ Programs could also offer opportunities for residents to recover from periods of exhaustion with post-call relief policies and create dedicated time for research and study. Other interventions that may improve burnout could include mindfulness and time management training through facilitated small group sessions. ${ }^{34}$ Despite the high rate of burnout among ophthalmology residents, 
Table 6 Most commonly listed factors that positively and negatively affect well-being (number of times cited)

\begin{tabular}{|l|l|}
\hline Factors that positively influence well-being & Factors that negatively influence well-being \\
\hline 1. Family, friends, and other nurturing relationships (194) & 1. Sleep deprivation and/or disruption (74) \\
\hline 2. Physical activity (129) & 2. Call duties (69) \\
\hline 3. Co-resident support (70) & 3. Work obligations and workload (59) \\
\hline 4. Healthy diet/Access to food (64) & 4. Lack of rest/leisure time (47) \\
\hline 5. Time in nature and the outdoors (57) & 5. Work hours (35) \\
\hline 6. Sleep (45) & 6. Stress of studying/lack of study time (33) \\
\hline 7. Time off (43) & 7. Poor faculty interaction and lack of support (31) \\
\hline 8. Support from faculty (32) & 8. Outside research, work, volunteer obligations (20) \\
\hline 9. Church, prayer, and spirituality (29) & 9. Poor diet (16) \\
\hline 10. Hobbies (27) & 10. Lack of exercise (16) \\
\hline
\end{tabular}

Table 7 Comparison of previous burnout studies in other specialties

\begin{tabular}{|c|c|c|c|c|c|c|c|c|c|c|}
\hline Study & Specialty & $\begin{array}{l}\text { No. of } \\
\text { responses } \\
\text { (response } \\
\text { rate) }\end{array}$ & Cohort & $\begin{array}{l}\text { Mean } \\
\text { EE } \\
\text { score }\end{array}$ & $\begin{array}{l}\text { \% with } \\
\text { high } \\
\text { EE } \\
\text { score }\end{array}$ & $\begin{array}{l}\text { Mean } \\
\text { DP } \\
\text { score }\end{array}$ & $\begin{array}{l}\% \text { with } \\
\text { high } \\
\text { DP } \\
\text { score }\end{array}$ & $\begin{array}{l}\text { Mean } \\
\text { PA } \\
\text { score }\end{array}$ & $\begin{array}{l}\text { \% with } \\
\text { low } \\
\text { PA } \\
\text { score }\end{array}$ & $\begin{array}{l}\% \text { with } \\
\text { all } \\
\text { categories } \\
\text { of high } \\
\text { burnout }\end{array}$ \\
\hline Becker et al ${ }^{17}$ & OB-GYN & 125 (29\%) & 23 programs & & 50 & & 32 & & 49 & 7 \\
\hline Golub et $\mathrm{al}^{20}$ & Otolaryngology & $684(50 \%)$ & National & 22.4 & 33 & 10.7 & 53 & 38 & 48 & 10 \\
\hline $\begin{array}{l}\text { de Oliveira } \\
\text { et } \mathrm{al}^{6}\end{array}$ & Anesthesia & 1508 (54\%) & National & $25^{\mathrm{a}}$ & & $8^{a}$ & & $38^{a}$ & & \\
\hline $\begin{array}{l}\text { Kimo } \\
\text { Takayesu } \\
\text { et al }\end{array}$ & $\begin{array}{l}\text { Emergency } \\
\text { Medicine }\end{array}$ & 218 (75\%) & 8 programs & & 33 & & 59 & & 59 & \\
\hline Elmore et $\mathrm{al}^{11}$ & General Surgery & 665 (NR) & National & & 57 & & 50 & & 16 & 10 \\
\hline $\begin{array}{l}\text { Guenette } \\
\text { and Smith }\end{array}$ & Radiology & 96 (19\%) & 20 programs & 24.3 & 37 & 10.6 & 48 & 33 & 40 & \\
\hline Ramey et $a^{22}$ & Radiation & 205 (28\%) & National & 20.5 & 28 & 7.1 & 17 & 39.4 & 12 & \\
\hline $\begin{array}{l}\text { Attenello } \\
\text { et } \mathrm{al}^{21}\end{array}$ & Neurosurgery & $346(21 \%)$ & National & & 43 & & 60 & & 36 & \\
\hline This study & Ophthalmology & 267 (23\%) & National & 27.9 & 55 & 11.8 & 46 & 38.8 & 13 & 8 \\
\hline
\end{tabular}

Abbreviations: EE, emotional exhaustion; DP, depersonalization; PA, personal accomplishment; NR, not reported.

a Median score instead of mean.

they are still satisfied with their specialty choice. Given the impact burnout can have on resident depression, wellness, and patient care, it is important to recognize its presence among the majority of ophthalmology residents and to encourage measures that promote well-being. It will also be important for future studies to identify interventions that could potentially improve the rate of burnout. As the nextgeneration ophthalmologists are trained, investing in strategies to cope with burnout may foster career-long work satisfaction and improve physician well-being and patient care.

\section{Funding}

This study was supported by an unrestricted departmental grant from Research to Prevent Blindness. The funding organization had no role in the design or conduct of this research.
Conflict of Interest

None declared.

\section{References}

1 Maslach C, Jackson SE, Leiter PM, Schaufeli WB. Maslach Burnout Inventory. 3rd ed. Palo Alto, CA: Consulting Psychologists Press; 1996

2 Lebensohn P, Dodds S, Benn R, et al. Resident wellness behaviors: relationship to stress, depression, and burnout. Fam Med 2013;45 (08):541-549

3 Bianchi R, Schonfeld IS, Laurent E. Burnout-depression overlap: a review. Clin Psychol Rev 2015;36:28-41

4 Schonfeld IS, Bianchi R. Burnout and depression: two entities or one? J Clin Psychol 2016;72(01):22-37

5 Wurm W, Vogel K, Holl A, et al. Depression-burnout overlap in physicians. PLoS One 2016;11(03):e0149913

6 de Oliveira GS Jr, Chang R, Fitzgerald PC, et al. The prevalence of burnout and depression and their association with adherence to 
safety and practice standards: a survey of United States anesthesiology trainees. Anesth Analg 2013;117(01):182-193

7 Shanafelt TD, Balch CM, Bechamps G, et al. Burnout and medical errors among American surgeons. Ann Surg 2010;251(06): 995-1000

8 Shanafelt TD, Bradley KA, Wipf JE, Back AL. Burnout and selfreported patient care in an internal medicine residency program. Ann Intern Med 2002;136(05):358-367

9 Block L, Wu AW, Feldman L, Yeh HC, Desai SV. Residency schedule, burnout and patient care among first-year residents. Postgrad Med J 2013;89(1055):495-500

10 West CP, Huschka MM, Novotny PJ, et al. Association of perceived medical errors with resident distress and empathy: a prospective longitudinal study. JAMA 2006;296(09):1071-1078

11 Elmore LC, Jeffe DB, Jin L, Awad MM, Turnbull IR. National survey of burnout among US general surgery residents. J Am Coll Surg 2016;223(03):440-451

12 Doan-Wiggins L, Zun L, Cooper MA, Meyers DL, Chen EH. Practice satisfaction, occupational stress, and attrition of emergency physicians. Wellness Task Force, Illinois College of Emergency Physicians. Acad Emerg Med 1995;2(06):556-563

13 Tran EM, Scott IU, Clark MA, Greenberg PB. Assessing and promoting the wellness of united states ophthalmology residents: a survey of program directors. J Surg Educ 2018;75(01):95-103

14 Shanafelt TD, Hasan O, Dyrbye LN, et al. Changes in burnout and satisfaction with work-life balance in physicians and the general US working population between 2011 and 2014. Mayo Clin Proc 2015;90(12):1600-1613

15 Dyrbye LN, West CP, Satele D, et al. Burnout among U.S. medical students, residents, and early career physicians relative to the general U.S. population. Acad Med 2014;89(03):443-451

16 Kimo Takayesu J, Ramoska EA, Clark TR, et al. Factors associated with burnout during emergency medicine residency. Acad Emerg Med 2014;21(09):1031-1035

17 Becker JL, Milad MP, Klock SC. Burnout, depression, and career satisfaction: cross-sectional study of obstetrics and gynecology residents. Am J Obstet Gynecol 2006;195(05):1444-1449

18 McNeeley MF, Perez FA, Chew FS. The emotional wellness of radiology trainees: prevalence and predictors of burnout. Acad Radiol 2013;20(05):647-655

19 Guenette JP, Smith SE. Burnout: prevalence and associated factors among radiology residents in New England with comparison against United States resident physicians in other specialties. AJR Am J Roentgenol 2017;209(01):136-141

20 Golub JS, Weiss PS, Ramesh AK, Ossoff RH, Johns MM III. Burnout in residents of otolaryngology-head and neck surgery: a national inquiry into the health of residency training. Acad Med 2007;82 (06):596-601
21 Attenello FJ, Buchanan IA, Wen T, et al. Factors associated with burnout among US neurosurgery residents: a nationwide survey. J Neurosurg 2018; February 9 (online ahead of print); doi: 10.3171/2017.9.JNS17996

22 Ramey SJ, Ahmed AA, Takita C, Wilson LD, Thomas CR Jr, Yechieli R. Burnout evaluation of radiation residents nationwide: results of a survey of united states residents. Int J Radiat Oncol Biol Phys 2017;99(03):530-538

23 Baer TE, Feraco AM, Tuysuzoglu Sagalowsky S, Williams D, Litman HJ, Vinci RJ. Pediatric resident burnout and attitudes toward patients. Pediatrics 2017;139(03):2163

24 Olson SM, Odo NU, Duran AM, Pereira AG, Mandel JH. Burnout and physical activity in Minnesota Internal Medicine resident physicians. J Grad Med Educ 2014;6(04):669-674

25 Pulcrano M, Evans SR, Sosin M. Quality of life and burnout rates across surgical specialties: a systematic review. JAMA Surg 2016; 151(10):970-978

26 Fletcher KE, Reed DA, Arora VM. Patient safety, resident education and resident well-being following implementation of the 2003 ACGME duty hour rules. J Gen Intern Med 2011;26(08): 907-919

27 Visser PS, Krosnick JA, Marquette J, Curtin M. Mail surveys for election forecasting? An evaluation of the Colombia Dispatch Poll. Public Opin Q 1996;60(02):181-227

28 Holbrook A, Krosnick J, Pfent A. The causes and consequences of response rates in surveys by the news media and government contractor survey research firms. In: Lepkowski JM, Tucker NC, Brick JM, et al, eds. Advances in Telephone Survey Methodology. New York, NY: Wiley; 2007

29 Mealing NM, Banks E, Jorm LR, Steel DG, Clements MS, Rogers KD. Investigation of relative risk estimates from studies of the same population with contrasting response rates and designs. BMC Med Res Methodol 2010;10:26

30 Ishak WW, Lederer S, Mandili C, et al. Burnout during residency training: a literature review. J Grad Med Educ 2009;1(02): 236-242

31 Salles A, Cohen GL, Mueller CM. The relationship between grit and resident well-being. Am J Surg 2014;207(02):251-254

32 Chaukos D, Chad-Friedman E, Mehta DH, et al. Risk and resilience factors associated with resident burnout. Acad Psychiatry 2017; 41(02):189-194

33 Kassam A, Horton J, Shoimer I, Patten S. Predictors of well-being in resident physicians: a descriptive and psychometric study. J Grad Med Educ 2015;7(01):70-74

34 West CP, Dyrbye LN, Rabatin JT, et al. Intervention to promote physician well-being, job satisfaction, and professionalism: a randomized clinical trial. JAMA Intern Med 2014;174(04): 527-533 